\title{
ORIGINAL ARTICLE \\ Chondroitinase administration and pcDNA3.1-BDNF-BMSC transplantation promote motor functional recovery associated with NGF expression in spinal cord-transected rat
}

\author{
L-L Xiong ${ }^{1}$, Y Li ${ }^{2}$, F-F Shang ${ }^{1}$, S-W Chen ${ }^{3}$, H Chen ${ }^{3}$, S-M Ju ${ }^{3}$, Y Zou ${ }^{1}$, H-L Tian ${ }^{3}$, T-H Wang ${ }^{1,2,4}$, C-Z Luo ${ }^{1,4}$ \\ and $\mathrm{X}-\mathrm{Y}$ Wang ${ }^{3,4}$
}

Study design: We evaluated whether combination of chondroitinase (chABC) administration and brain-derived neurotrophic factor (BDNF)-mesenchymal stem cell (MSC) transplantation could provide an optimal effect for the treatment of spinal cord injury (SCI) subjected to complete transection.

Objectives: Behavioral assessments and DBA tracing were used to evaluate the effects of combination of chABC administration and BDNF-MSC transplantation on axonal regeneration and functional improvement in SCT rats.

Setting: Sichuan, China

Methods: Bone mesenchymal stem cells (BMSCs) were cultured and overexpressed BDNF recombinant vector was constructed into MSCs, then transplanted into the impaired spinal cord, together with chABC administration. Finally, the cortical spinal tract regeneration was detected by DBA tracing at 4 weeks post operation, and the expression of nerve growth factor (NGF), BDNF, neurotrophic factor (NT)-3, NT-4, fibroblast growth factor (FGF-2)-2, B cell lymphoma 2 (BCL-2) assaciated X protein (BAX) and BCL-2 in the caudal cord tissues was assessed by reverse transcription-PCR.

Results: Animals received both BDNF-BMSC transplantation and chABC administration presented the best functional recovery and obvious axonal regeneration. Moreover, NGF expression was significantly higher than that in the other groups.

Conclusion: Co-treated strategy could effectively promote motor functional recovery and axonal regeneration in SCT rats associated with NGF upregulation.

Spinal Cord (2016) 54, 1088-1095; doi:10.1038/sc.2016.55; published online 28 June 2016

\section{INTRODUCTION}

Spinal cord injury (SCI) is a kind of myeleterosis in different degrees caused by acute injury, chronic damage or trauma. The annual morbidity of SCI is $12.1-57.8$ per million people in Western Europe and 25.2-52.5 per million people in America. ${ }^{1}$ Patients subjected to SCI result in motor and sensory dysfunction and disability, which brings out heavy burden to family and society.

Apoptosis and necrosis of cells will be caused by primary damage and secondary damages including oxygen radical, lipid peroxidation and inflammation after SCI. The formation of vast glia scar and cavitation in the lesion area restrained the regeneration of axons and impede the restoration of neural function. It has been well known that scar, an important factor, can inhibit the axonal regeneration and extension, in which the upregulated expression of chondroitin sulfate proteoglycans, a strong axon regeneration inhibitor molecule in extracellular matrix in the injured area, is the main functional element. ${ }^{2,3}$ Chondroitinase (chABC) can rupture the most important restraining factor, GAGS, in chondroitin sulfate proteoglycans to promote the functional recovery. ${ }^{4-6}$ Therefore, chABC administration may help the axonal growth in the injured area after SCI.

It is well known that the interruption of the signal pathway following nerve injury after SCI underlies the reason of dysfunction, whereas material from cellular or tissue engineering may provide effective scaffold for nerve regeneration. Mesenchymal stem cells (MSCs), as a kind of multipotent stem cells in mesoderm, could be considered as the bridge for nerve growth. The other reason is that bone mesenchymal stem cells (BMSCs) could be easily isolated from bone marrow, muscle, bone, cartilage, fat and vessel, and they could differentiate into many kinds of cells to compose various tissues such as bone, cartilage, fat, tendon and nerve. ${ }^{7-9}$ Therefore, BMSCs possessed several advantages that are beneficial for transplantation. When transplanted to the lesion sites of spinal cord, MSCs could differentiate into neural-like cells or gliocyte-like cells and secrete several neurotrophic factors (NTFs) to promote axonal regeneration

\footnotetext{
${ }^{1}$ Department of Anesthesiology, Institute of Neurological Disease, Translational Neuroscience Center, West China Hospital, Sichuan University, Chengdu, People's Republic of China; ${ }^{2}$ Department of Neurosurgery, Institute of Neuroscience, Nol. Affiliated hospital, Kunming Medical University, Kunming, People's Republic of China and ${ }^{3}$ Department of Neurosurgery, Shanghai Jiao Tong University Affiliated 6th People's Hospital, Shanghai, People's Republic of China

${ }^{4}$ These authors contribute equally to this work.

Correspondence: Professor T-H Wang or Professor C-Z Luo, Department of Anesthesiology, Institute of Neurological Disease, Translational Neuroscience Center, West China Hospital, Sichuan University, Chengdu 610041, People's Republic of China.

E-mail: tinghua_neuron@263.net or chaozhil@aliyun.com

or Professor X-Y Wang, Department of Neurosurgery, Shanghai Jiao Tong University Affiliated 6th People's Hospital, Shanghai 200233, People's Republic of China.

E-mail: 851794319@qq.com

Received 15 December 2014; revised 16 January 2016; accepted 3 March 2016; published online 28 June 2016
} 
and remyelination, ${ }^{10}$ which is associated with motor function repair. ${ }^{10}$ Brain-derived neurotrophic factors (BDNFs) purified from pig brain in 1982 , with the molecule weight $12.3 \mathrm{kDa}$ and isoelectric point 10 , share the function in maintaining neural survival and neurite outgrowth. ${ }^{11}$ It has been known that overexpression of BDNF in the lesion area could prevent the infection and secondary damage. ${ }^{12}$ Together, BMSCs combined with growth factor administration may strengthen the positive effect for functional recovery. In this study, we test the following hypothesis: whether chABC administration combined with BDNF-MSC transplantation may provide an optimal strategy for the treatment of SCI. Then, we explore the underlying mechanism involving in nerve growth factor (NGF).

\section{MATERIALS AND METHODS}

\section{Isolation and culture of BMSCs}

Adult rats were killed with anesthesiology and sterilized for $3 \mathrm{~min}$ in $75 \%$ ethanol. Then the femur was aseptically taken out and the ends were opened. This was followed by a washing in the bone marrow cavity with culture solution for three times, and the cell suspension was collected and filtered using 200-mesh syringe or 4-G needle, then seeded into the bottle and cultured in an incubator with $5 \% \mathrm{CO}_{2}$. On the 5th day, the culture solution was replaced half, than replaced completely per 3 days. After the growth and morphology of cells were observed, cells at 5 passages were harvested to perform subsequent experiment including cell transfection and immunohistochemistry staining.

\section{Cloning eukaryotic vector of pcDNA3.1-BDNF}

Total RNA was extracted from the rat brain according to the introduction of the TRIzol RNA Kit (Takara Bio Inc., Otsu, Japan). Then, the PrimeScript II 1st Strand cDNA Synthesis Kit (TaKaRa Biotechnology, Dalian, China) was used for reverse transcription from mRNA to cDNA. BDNF mRNA was amplified by using the primer designated as upstream primer (HindIII) - $5^{\prime}$-CCCAA GCTTGCCACCATGACCATCCTTTTCCTTACT-3' - and downstream primer (EcoRI) - 5'-CGCGGATCCTTTCTTCCCCTTTTAATGGTCA-3', by using the Long Range PCR Kit (Sangon Biotech, Shanghai, China). Finally, the products of BDNF were detected by agarose gel, collected and isolated, and then digested with iHind III and EcoR I at cleavage sites of the 5' end of the primer, upstream and downstream, respectively. On the other hand, pcDNA3.1 plasmid was digested by HindIII and EcoRI, and then coupled with target DNA by T4 DNA ligase in $16^{\circ} \mathrm{C}$ for $20 \mathrm{~h}$. The products of reaction were transfected to colibacillus DH5 $\alpha$ and inoculated into the medium containing ampicillin, which helped in screening the recombined plasmid. Finally, HindIII and EcoRI were used to identify the recombinant plasmid.

\section{PcDNA3.1-BDNF transfected MSCs}

When the MSCs covered over $60 \%$ of the surface of the culture dish, all the culture solution was replaced and processed the transfection. The purified pcDNA3.1-BDNF plasmid and naive plasmid were, respectively, transfected into MSCs. When the cells covered over $60 \%$ of the surface of the culture dish, the culture solution was replaced with G418 solution to screen the cells. Finally, the positive cells screened by G418 were collected to test the expression of BDNF by anti-BDNF via immunohistochemical staining. To detect the activity of BDNF in MSCs, cortical neurons from neonatal rats were cultured, and the supernatant of BDNF-MSC or MSC was only added into the medium (amount to one-third cultured medium), and the cell neurite elongation and number were counted. In order to trace the morphology of MSCs, in vivo cultured MSCs were also labeled with HSV-GFP, routinely.

\section{Animal and grouping}

Adult female rats, weighing $200 \pm 20 \mathrm{~g}$, were individually housed in a quiet and non-strong light vivarium with free access to water and food. Sixty rats were divided into 5 groups, shown in Table 1 .
Table 1 Animal grouping for experiment

\begin{tabular}{lccccc}
\hline & Sham & SCT & SCT+chABC & $\begin{array}{c}\text { SCT+ BDNF- } \\
\text { MSCS }\end{array}$ & $\begin{array}{c}\text { SCT+chABC } \\
+ \text { BDNF-MSCS }\end{array}$ \\
\hline Behavioral test & 8 & 8 & 8 & 8 & 8 \\
Morphology & 5 & 5 & 5 & 5 & 5 \\
RT-PCR & 7 & 7 & 7 & 7 & 7 \\
\hline
\end{tabular}

Abbreviations: BDNF, brain-derived neurotrophic factor; chABC, chondroitinase; MSC, mesenchymal stem cell; RT-PCR, reverse transcription PCR.

\section{Animal model and locomotor function assessment}

The rats were anesthetized intraperitoneally by $2 \%$ pentobarbital sodium $30 \mathrm{mg} \mathrm{kg}^{-1}$. T10 was located by counting the number of back ribs using hand touching. A midline incision was made in the back following skin prepared, and then the skin and subcutis were exposed, paravertebral muscles were decoherenced and spinal process and vertebral plate were eliminated. Eventually, the spinal cord was completely transected at the T10 vertebra with the help of microscissors. In order to ensure the completeness of the transection, the spinal transection should be assured by lifting the cut ends by small forceps. Subsequently, the spinal cord has been completely transected and there is still no tissues remaining. Dura has also been transected. For cell transplantation, MSC suspension $\left(6 \times 10^{5}\right.$ per $\left.10 \mu \mathrm{l}\right)$ was injected into the caudal cord below the lesion $(1.0 \mathrm{~cm})$ in the cell transplanted group. Simultaneously, $6 \mu \mathrm{l}$ of chABC $\left(1 \mu \mathrm{ml}^{-1}\right)$ was pumped into the spinal cord of rats using micropump after surgery for every 2 days until 1 week before drawing materials.

The test for the Basso, Beattie and Bresnahan locomotor rating scale was performed at 2000 to 2100 hours, 1, 7, 14 and 28 days post operation to assess the hindlimb locomotor function by three investigators that were blind to the subject's experimental treatment.

\section{BDA anterograde tracing}

At 14 days post operation, the rats were anesthetized intraperitoneally by $2 \%$ pentobarbital sodium and fixed in stereotaxic head-holder device; the skull was exposed and the sagittal suture and the coronal suture were verified. A rectangle bone flap, $2 \times 8 \mathrm{~mm}$, was made in the area $2 \mathrm{~mm}$ beyond sagittal suture, $4 \mathrm{~mm}$ to coronal suture. Ten percent solution of biotin dextran amine (BDA) was microinjected into four sites at the depth of $1.5 \mathrm{~mm}$ through the bone flap $(0.5 \mu \mathrm{l}$ per site), and the needle was maintained for $5 \mathrm{~min}$ to avoid the solution overflowing. After injection, the scalp was sutured. Finally, the rats were properly settled and kept in a warm place with free access to food and water. Two weeks after survival, the rats were killed and the caudal spinal cords were harvested and then subjected to horseradish peroxidase-conjugated enzyme staining to show the distribution of BAD-positive fibers from three fields (400X) in each section, with three sections from each animal. Furthermore, in order to further illustrate the axon regeneration and show it with the grafted GFP-labeled MSC clearly, we performed BDA-cy3 red immunofluorescent staining. Briefly, this step was the same as the above processes, except for being performed using 1:400 StrePtavidin-cy3, and then the images were observed under the fluorescent microscope (Leica, Wetzlar, Germany).

\section{RT-PCR}

RT-PCR was used to determine the expression level of many NTFs (NGF, BDNF, IGF-1, BFGF, B cell lymphoma 2 (BCL-2) assaciated X protein (BAX) and BCL-2) on the side of the transplanted spinal cord of host. Total RNA was isolated from the spinal cord tissues on the side of the transplant by using TRIzol (Invitrogen, Carlsbad, CA, USA), according to the manufacturer's instruction. cDNA was generated using the Revert Aid First Strand cDNA Synthesis Kit (Thermo, Waltham, MA, USA). This was followed by further PCR amplification for each cDNA sample, using rat-specific primers by CFX-96 (Bio-Rad, Hercules, CA, USA).

\section{Western blot}

Cultured MSCs with BDNF transfection and control were harvested, and then lysed at $4{ }^{\circ} \mathrm{C}$ in a RIPA lysis buffer (Beyotime, Jiangsu, China). The supernatant 

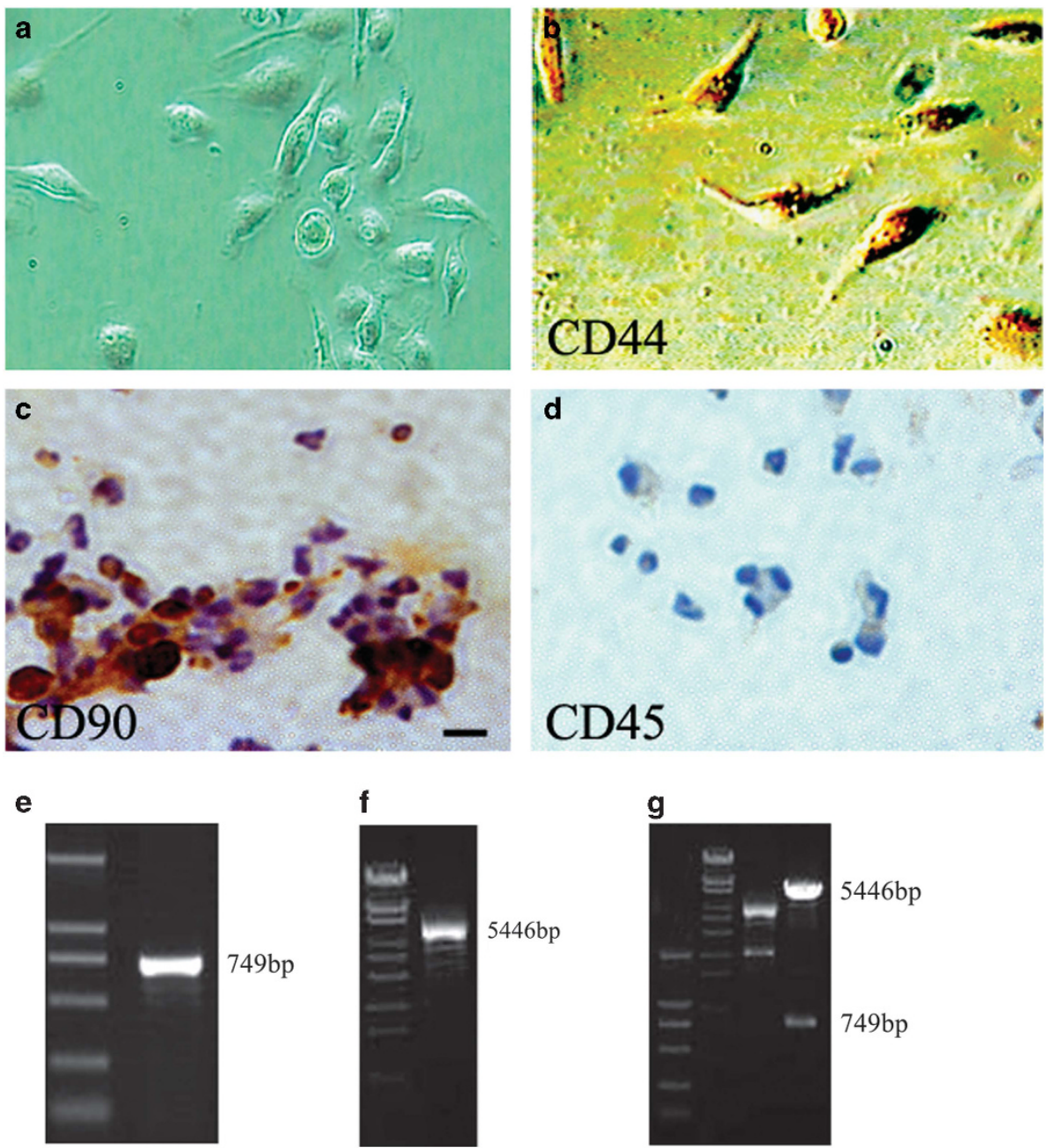

Figure 1 Identification of the cultured MSCs and pcDNA3.1-BDNF-MSCs vector clone. Cultured BMSC at 5th generation is shown in a. Brown staining by anti-CD44 (98\%) and anti-CD90 (95\%) exhibited in the cytoplasm is shown in b and c. (d) Image shows anti-CD45 detection (0\%), which gives a negative staining. Scale bar $=50 \mu \mathrm{m}$. (e) Products of BDNF-cDNA by RT-PCR were shown by $1 \%$ agarose gel. (f) The empty plasmid of pcDNA3.1 was cloned and shown by $1 \%$ agarose gel. (g) The products of recombinant plasmid double digested by HindIII and EcoR I were shown by agarose gel. Scale bar $=50 \mu \mathrm{m}$, shown in $\mathbf{c}$.

was obtained after centrifuging at $8000 \mathrm{~g}$ for $10 \mathrm{~min}$ at $4{ }^{\circ} \mathrm{C}$ and stored at $-80^{\circ}$ $\mathrm{C}$ for later use. The protein concentration of supernatant was determined with bicinchoninic acid (BCA) assay. Samples (50 $\mu$ g per lane) blended with buffer were loaded on to each lane and electrophoresed on SDS-PAGE (3\% spacer gel, $12 \%$ separation gel) then transferred to a poly(vinylidene fluoride) membrane. Then, primary antibody for BDNF (1:1000, Abcam, Cambridge, MA, USA) and $\beta$-actin (1:5000, Abcam) was used to incubate poly(vinylidene fluoride) membrane at $4{ }^{\circ} \mathrm{C}$ overnight, and a horseradish peroxidase-conjugated goat anti-rabbit IgG at room temperature for $30 \mathrm{~min}$ was used by routing western blot (WB) protocol. Finally, the membrane was put into the ECL Western blotting kit (Alpha Innotech, Biorad Laboratories, Berkeley, CA, USA) for $1 \mathrm{~min}$ and then imaged using the Imagining System (Alpha Innotech). Then, densitometry analysis was performed by the Quantity One Imagining System. $\beta$-Actin was used as an internal control (Alpha Innotech).

\section{Statistical analysis}

All date were expressed as the mean \pm s.e.m. The variance analysis and the $T$-test were performed by the SPSS 17.0 software package (SPSS, IBM Corporation, New York, NY, USA). Statistical significance was defined as $P<0.05$.

\section{RESULTS}

\section{MSCs culture}

The primary cultured MSCs presented heterogeneous morphology with square or spindle shape. The parenchyma cells could be wiped out by subculture. The 5th generation cells were almost purified to MSCs, with long fusiform morphology, strong refractivity, clear boundary and plump nucleus in the center (Figure 1a). These cells ranked in racial pattern or swirling, which showed directionality. Immunostaining showed that MSCs expressed CD44 (98\%) and CD90 (95\%), but not CD45 (0\%), confirming that they were BMSCs (Figures 1b-d).

\section{Clone of BDNF gene and construction of eukaryotic vector of pcDNA3.1-BDNF}

The production of RT-PCR, BDNF-cDNA, showed bends of 749 bp by $1 \%$ agarose gel electrophoresis, corresponding to the expected result. The BDNF-cDNA was harvested from agarose gel and then purified with column for later use. Construction of recombinant plasmid pcDNA3.1 was prepared and digested by HindIII and EcoRI, and then target BDNF sequence was added. By using linked enzyme, 

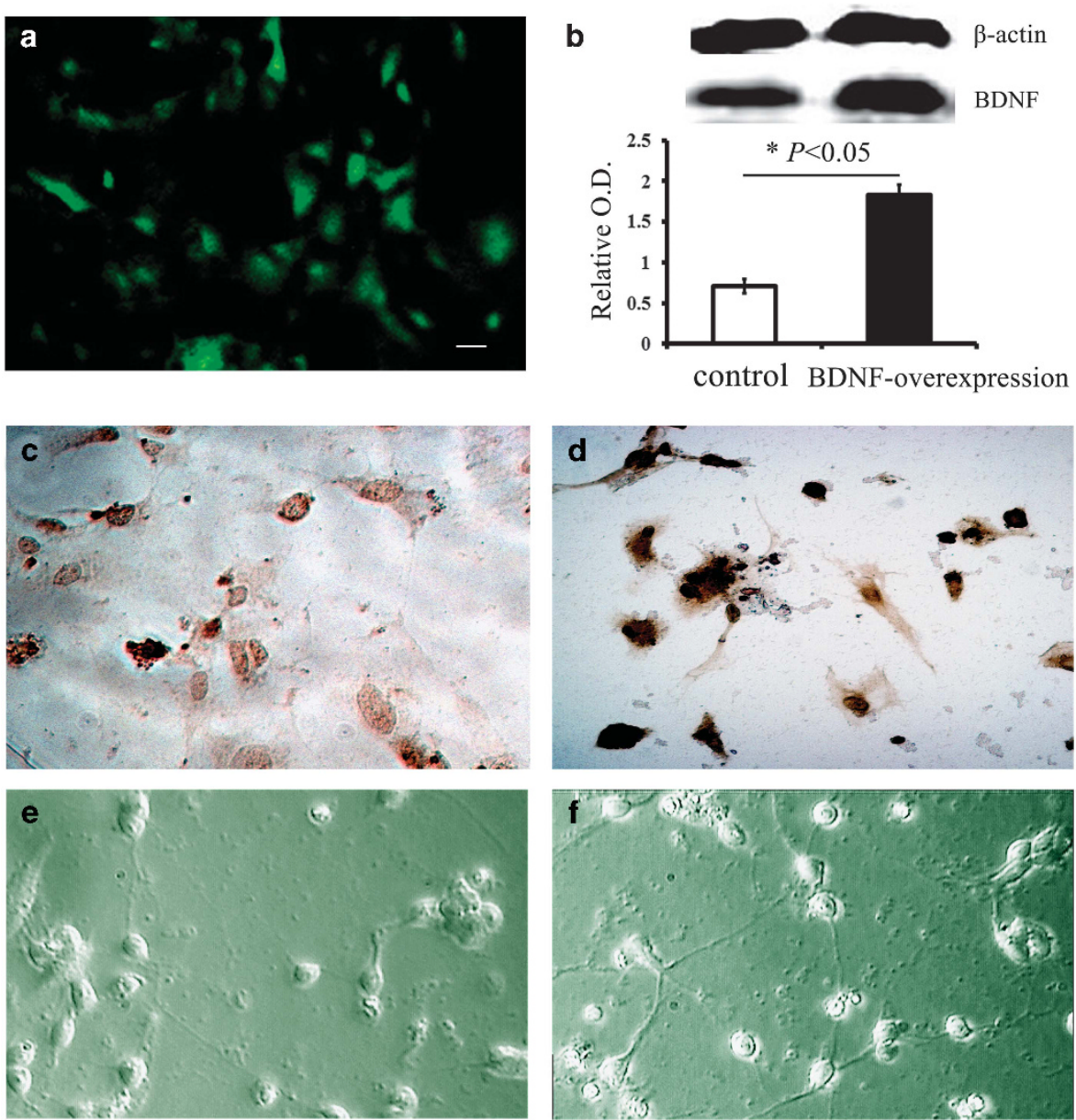

Figure 2 BDNF expression vector was transfected to MSCs successfully. (a) Transfected MSCs screened by G418 and GFP fluorescence were seen. (b) Expression of BDNF was exhibited in the BDNF transfection group and the control group, indicated by western blot. (c, d) Immunocytochemical staining showed that expression of BDNF in the transfection group (d) was higher than that in the control group (c). (e, f) The cell supernatant of MSCs (e) or BDNFMSCs (f) was used to culture the cortical neurons, respectively. The BDNF-MSC supernatant exhibited a good consequence in inducing neurite outgrowth in cultured cortical neurons. Scale bar $=50 \mu \mathrm{m}$, shown in a.

BDNF-cDNA was added into pcDNA3.1 vector. In order to identify correction, the recombinant plasmid showed segment of $749 \mathrm{bp}$ and another segment of $5.4 \mathrm{~kb}$ by agarose gel electrophoresis after double digested by HindIII and EcoRI, corresponding to the expected result (Figures 1e-g).

\section{Effect of recombinant pcDNA3.1-BDNF-transfected rat MSCs}

Twelve hours after transfection, cells that expressed BDNF could grow in the medium containing G418, whereas the cells that did not receive transfection died gradually. The labeled GFP-BMSCs emit green fluoresces under fluorescent microscope (Figure 2a). The expression of BDNF in the transection group was higher than that in the control group, confirmed by western blot (Figure 2b). Meanwhile, immunocytochemical staining showed that the cultured cells were BDNF positive (Figures $2 \mathrm{c}$ and $\mathrm{d}$ ). Moreover, supernatant from MSCs and BDNF-MSCs was administrated to cultured cortical neurons, which showed a significant increase on growth in BDNF administrated one than that in MSCs only (Figures 2e and f).

Behavioral detection after combination therapy with chABC and MSCs in SCT rats

BBB score in all groups showed 21 scores before operation, and the score of model group descended to 0 after surgery and then ascended in some degree. The score of the sham group was higher than in the SCI group. The individual treatment with chABC or MSCs could enhance the locomotor function. What is more, combination therapy with chABC and MSCs obtained highest scores (Figure 3a). At the end of the experimental time point, engrafted MSCs still survived in the host spinal cord, which was confirmed by GFP labeling (Figure 3b).

\section{BDA tracing}

First of all, we tested whether the regenerative fibers could penetrate the scar and reach below the lesion site, and all images were taken at $1 \mathrm{~cm}$ below the lesion. In the co-treated group, the extensive axons from transected spinal cord (CST) could be found at the cord above the lesion site (Figures $4 \mathrm{a}$ and $\mathrm{b}$ ), and there are regenerative axons penetrating the scar (Figure 4c). Moreover, there are few positive stain axons with spot or silk character that could be found in CST location, despite the number of fibers being only $\sim 10 \%$ of total (Figures $4 \mathrm{~d}, \mathrm{f}$ ). After detecting that the CST could regenerate a few axons to reach below the lesion site, we compared the number of axons in cord below the lesion site from each group. The Sham group presented significantly more BDA-positive staining fibers in the dorsal of white matter obtained through the lesion area and reached the caudal site of the spinal cord, compared with other transection groups. The individual treatment with $\mathrm{chABC}$ or MSCs showed similar number 


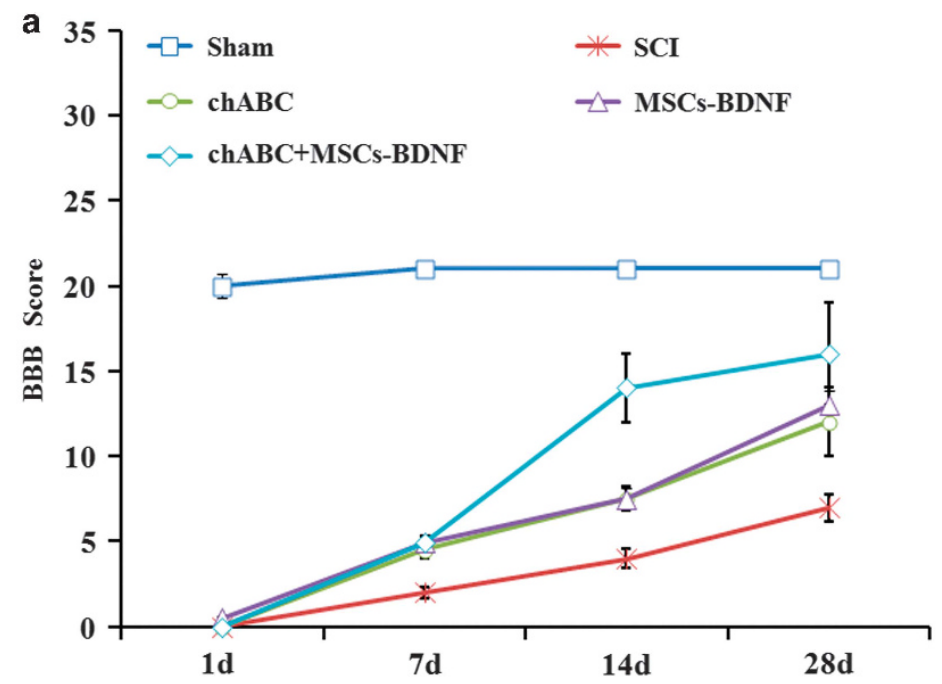

b
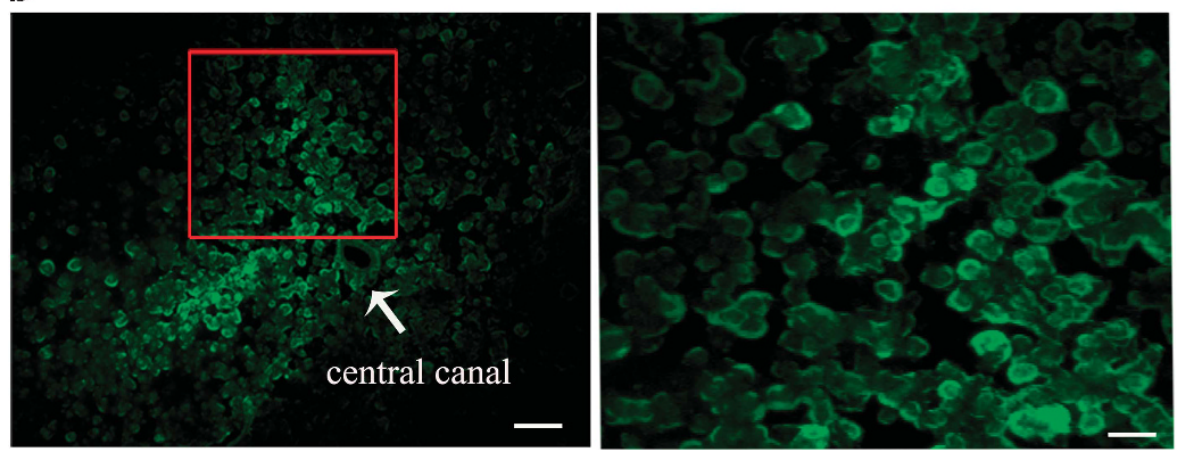

Figure 3 Combination therapy of chABC and MSCs significantly improved locomotor function, and engrafted MSCs survived in the host cord. (a) Mean value of the BBB score in different groups at different time (1, 7, 14 and 28 days post operation (dpo)) was shown in the sham group, the SCT group, the chABC group, the pcDNA3.1-BDNF-MSC group and the co-treatment group (value $=$ mean \pm s.e.m., $n=8, P<0.05$ ). (b) Engrafted MSCs could survive in the host spinal cord, confirmed by GFP labeling. Left: a low-power picture of the graft in a spinal cord section; the white arrow represents the central canal; right: a high-power picture of the cells from the left. Scale bar $=50 \mu \mathrm{m}$ in the left, $25 \mu \mathrm{m}$ in the right.

of fibers. In addition, BDA-cy3 labeling showed that the red-labeled axons regenerated from the host CST into the graft, axon growth through graft cells (identified by GFP staining), and then axons growing out of the graft back into host spinal cord at the caudal end of the graft (Figures 4g-i). Together, these showed that the BDA-positive staining fibers in the dorsal of white matter grew through the lesion area in the combination therapy group (Figure 5a-f).

\section{Expression of NTF}

To explore the mechanism after chABC and MSCs treatment in SCT rats, we identified the expression level of NTF, including 6 growth factors, in the side of the transplanted spinal cord of host. Of those 6 factors, 5 factors (BDNF, IGF-1, BFGF, BAX and BCL-2) showed no statistically significant difference between combination therapy group and other groups. However, the combined transplantation group presented a significant increase in the expression of NGF when compared with the BMSCs $(P=0.015)$ and $\operatorname{chABC} \operatorname{group}(P=0.018$; Figure 6).

\section{DISCUSSION}

The primary pathologic change following SCT was the bleeding of gray matter, cell apoptosis and necrosis of neurons ${ }^{13}$ in the spinal cord. The secondary pathologic change was the main reason that led to dysfunction of the spinal cord including ischemia of tissue, internal environment imbalances, oxygen radical and lipid peroxidation, inflammation excitatory toxicity and excitatory amino acid. ${ }^{14}$ Oxygen radical and lipid peroxidation, inflammation and excitatory toxicity all may result in more apoptosis and necrosis of cells. The formation of glial scar after SCI could prevent inflammation but impede axonal regeneration. Moreover, Lemons et al. found that chondroitin sulfate proteoglycans were the main substance in scar that can inhibit axon regeneration. ${ }^{2}$ Therefore, improved scar microenvironment may contribute later functional recovery.

In this study, we isolated the MSCs and constructed the eukaryotic expression vector pcDN3.1-BDNF and then transfected the pcDN3.1BDNF into BMSCs successfully. Moreover, compared with the separate application of exogenous chABC or pcDNA3.1-BDNF-MSC transplantation to rats, combination of exogenous chABC with pcDNA3.1-BDNF-MSC transplantation presented more axon regeneration, which then got through the injured area and reached partially to the caudal spinal cord tissue; subsequently, this may result in a better locomotor functional recovery in hindlimbs of SCI rats. The results therefore indicated that combination strategy appears better than alone. Previously, it demonstrated that chABC administration could enhance the recovery of hindlimb locomotor function. ${ }^{15}$ Our study also confirmed this addition of chABC. In addition, Brazelton 

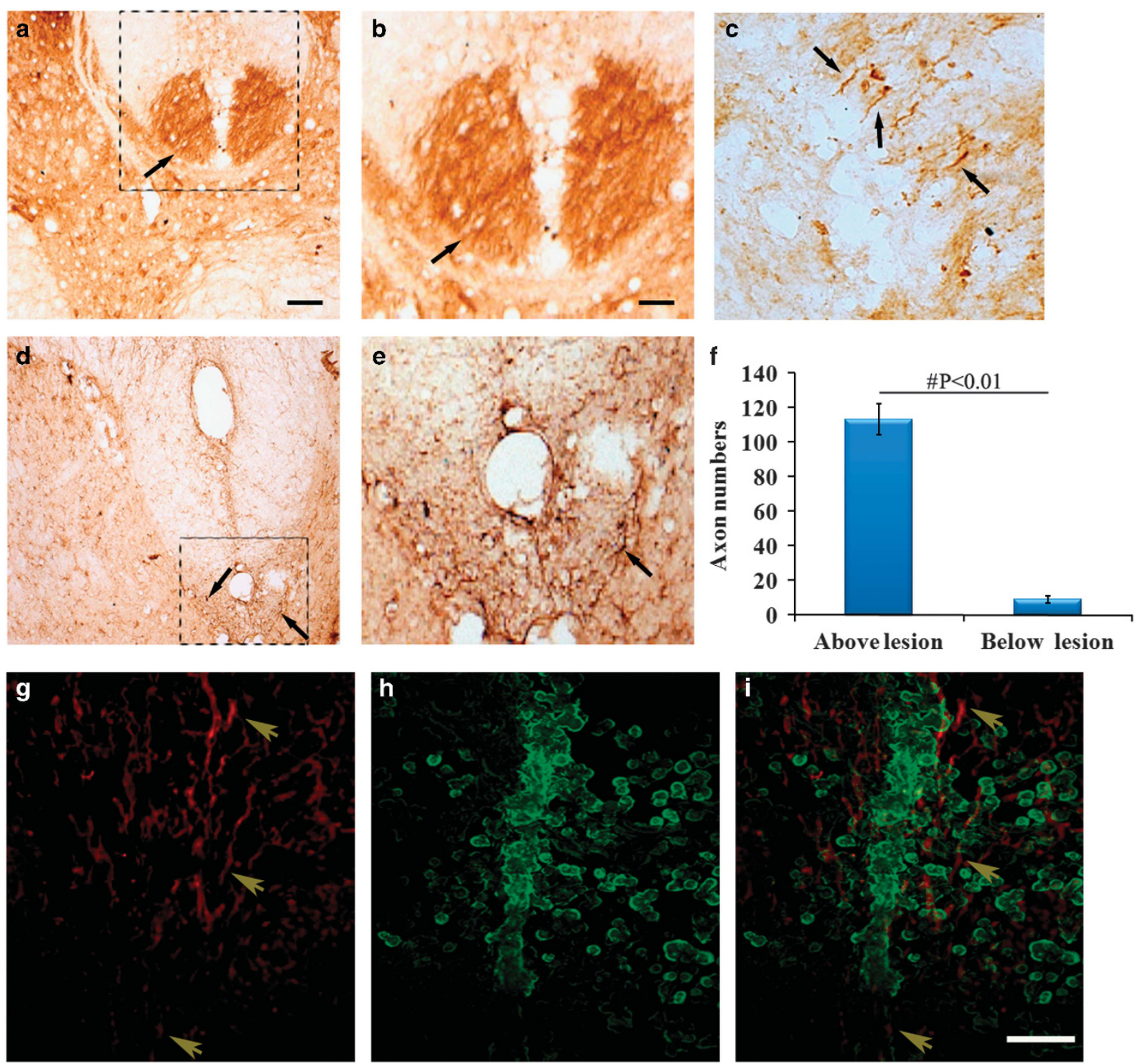

Figure 4 Exhibition of axon regeneration across the injury site in the co-treated group. BDA-positive fibers representing cortical spinal tract in above and below lesion are shown in $\mathbf{a}$ and $\mathbf{d}$, respectively (black arrow). (b, e) High-magnification pictures from $\mathbf{a}$ and $\mathbf{d}$ are shown. (c) The regenerative fiber could be observed in injury site. $\mathbf{f}$ amounts to the comparison analysis between above and below lesion. (g) BDA-cy3 immunofluorescent staining for the regenerated axons. (h) Engrafted MSCs in the host spinal cord, confirmed by GFP labeling. (i) Merged picture for the BDA-cy3 staining and the engrafted MSCs. The brown arrows represent the positive regenerated axons. Scale bar $=100 \mu \mathrm{m}$ in $\mathbf{a}, \mathbf{c}, \mathbf{d}$ and $50 \mu \mathrm{m}$ in $\mathbf{b}, \mathbf{e}, \mathbf{g}, \mathbf{h}, \mathbf{i}$.

et al. proved that the application of MSCs could promote hindlimb locomotor function recovery. ${ }^{16}$ Moreover, co-treatment with BDNF and MSCs increased hindlimb locomotor functional recovery in the SCI rats, when compared with separate application of BDNF or MSCs to the rats. ${ }^{17}$ In our study, combined exogenous chABC with pcDNA3.1-BDNF-MSC transplantation acquired better recovery and axon regeneration in the injured spinal cord, as compared with other groups. These fruitful findings may provide the optimal strategy for the repair of SCI.

chABC application could degrade the scar and promote the budding and regeneration of the injured neurons but could not in the integrated spinal cord. Bradbury et al. applied chABC to injured spinal cord by intrathecal injection and found that the chondroitin sulphate-glycosaminoglycan (CS-GAGs) in the lesion area were degraded. ${ }^{4}$ Yick et al. proved that application of chABC could enhance the regeneration of neuronal fibers in the damaged spinal cord, and no budding was found in the sham group. ${ }^{18}$ The result of our study showed that the SCI rats received chABC and exhibited more budding of axons than the control one subjected to injury only. BDNFs have an important role in the growth of the neural system and protection and reconstruction of the injured neural system. Goto et al. studied the expression of BDNF after SCI and found that the upregulation of BDNF is at 3 weeks after SCI, and the motor neurons were markedly stained by the BDNF antibody. The astrocyte-like cells were observably stained by the BDNF antibody in the gray matter 4 weeks after SCI, which showed that BDNF was associated with the repair mechanism ${ }^{19}$ in later stage. Diener et al. found that BDNF can be expressed long time in red nucleus in spinal cord hemisection rats. ${ }^{20}$ Meanwhile, 

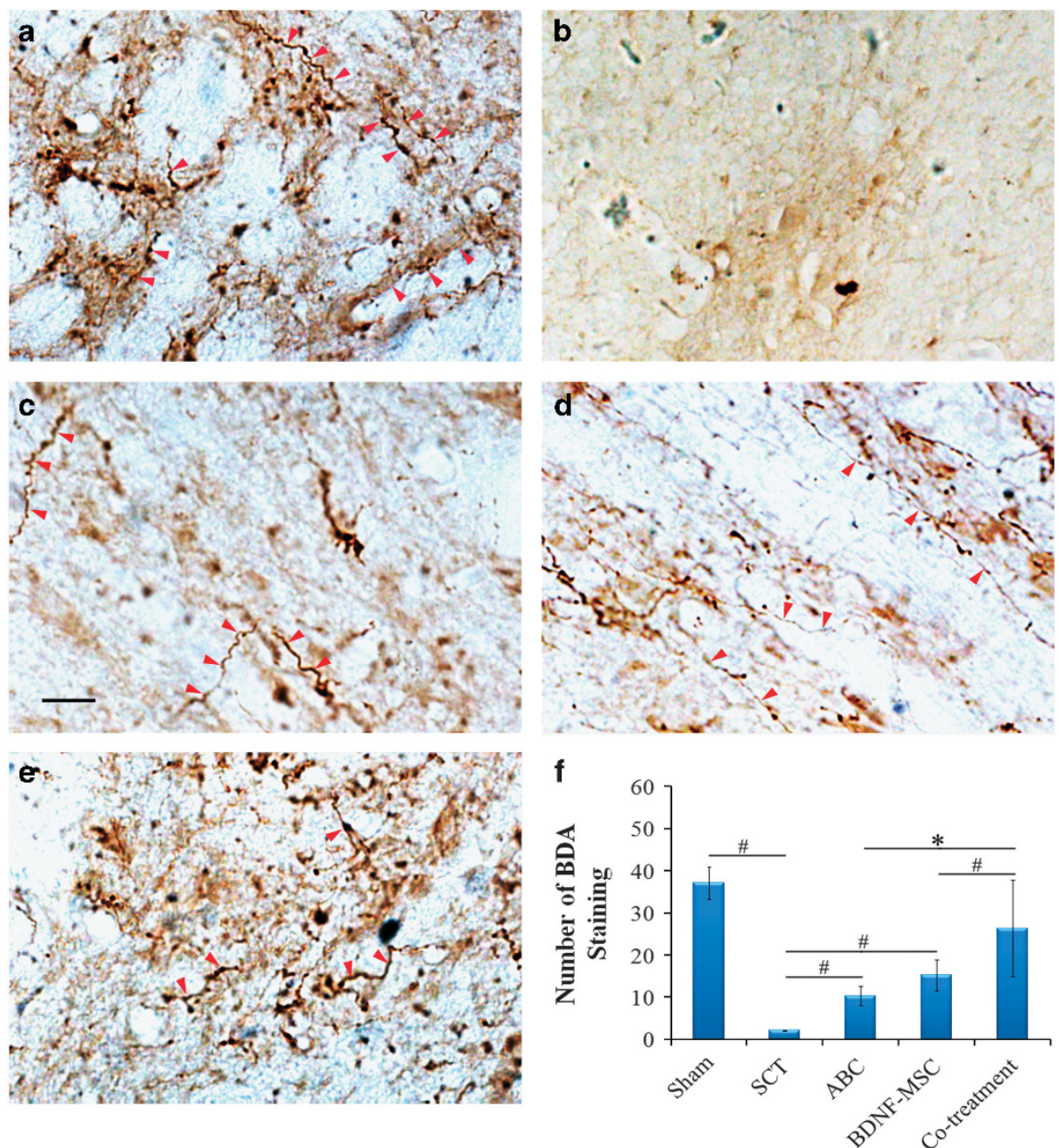

Figure 5 Results of BDA tracing in each group. The sections were selected from spinal cord segment caudal to the transection sites, and the pictures were taken at $1 \mathrm{~cm}$ below the lesion. BDA-positive axons are short brown silky strands in these pictures. The following pictures a-e show the section selected from the sham group, the SCT group, the chABC group, the pcDNA3.1-BDNF-MSC group and the co-treatment group, respectively. The quantitative results are shown in $\mathbf{f}$. \# compared each other, $P<0.05$. Scale bar $=100 \mu \mathrm{m}$, shown in $\mathbf{c}$.

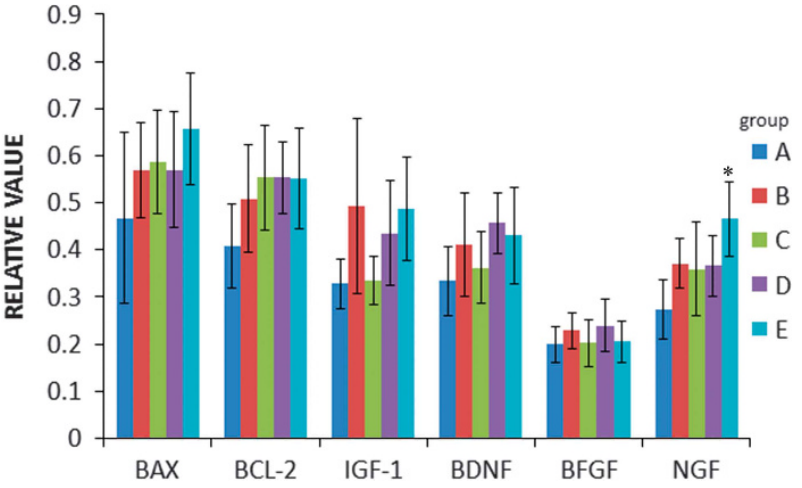

Figure 6 The quantity analysis of NTF expression. The expression of BAX, BCL-2, IGF-1, BDNF, BFGF and NGF on the side of the transplanted spinal cord in different groups was exhibited (value=mean \pm s.e.m., $n=5$, $P<0.05)$. NGF expression in the co-transplantation group was significantly upregulated as compared with the control one. ${ }^{*} P<0.05$, compared with the control one. A, sham group; $B, S C T$ group; $C$, chABC group; D, pcDNA3.1BDNF-MSC group; E, co-treatment group.
BDNF could increase the reactivity-specific spinal neurons subjected to chronic injury. ${ }^{21}$

MSCs could be as a cell bridge for carrying BDNF to promote the growth of axons. True blue chloride retrograde tracing was performed 3 months later and showed that true blue chloride was detected in the rostral thoracic spinal, red nucleus and sensory-motor cortex. ${ }^{22}$ However, Cizkova et al. injected hMSCs into rats intravenously 7 days after SCI and proved that hMSCs differentiated into oligodendroglia cells, and no NeuN-expressing cells were observed. However, the generated axons accumulated with GAP-43 were found in the injured white matter after transplantation. ${ }^{10}$

In our study, we focused on the role of MSCs that can carry BDNF. Moreover, BDNF-MSC transplantation could offer better effect to enhance the extend of axons compared with separate application of BDNF or MSCs. Sasaki et al. found that BDNF-hMSC transplantation presented more beneficial effect to the animals' motor functional recovery, and more corticospinal track neurons survival in $\mathrm{M} 1$, at 5 weeks after transplantation. ${ }^{23}$ Furthermore, the present study revealed that the survival of injured neurons and the regenerated axons increased markedly. About $10 \%$ of regenerated axons reached the caudal cord after injury, indicated by BDA tracing. These results 
do augment the idea that co-treatment based on BDNF-MSC and chABC could effectively promote the axons regeneration and functional repair. In molecular mechanism, the functional repair and axonal growth may be linked to NGF expression. NGF, one of the most important growth factor, has been well documented for its role on neurite growth. ${ }^{24,25}$ Our results exhibited new evidence to support that NGF is crucial for functional improvement in SCT rats. These results may help us understand the mechanism on co-treated strategy based on NGF for the treatment of SCI in future.

Together, this study provides the crucial evidence to understand the effect of chABC administration together with BDNF-MSC transplantation in promoting motor functional recovery, and the underlying mechanism is associated with the NGF expression in SCT rats. These findings described novel evidence that would be useful for the treatment of SCI in the future clinical trials.

\section{CONFLICT OF INTEREST}

The authors declare no conflict of interest.

\section{ACKNOWLEDGEMENTS}

This work was supported by grants from the National Natural Science Foundation of China (Grant No. 81271358 and No. 81070991) and the Natural Science Funding of Zhejiang Province (Grant Y2090864).

1 van den Berg ME, Castellote JM, Mahillo-Fernandez I, de Pedro-Cuesta J. Incidence of spinal cord injury worldwide: a systematic review. Neuroepidemiology 2010; 34 184-192 discussion 92.

2 Lemons ML, Howland DR, Anderson DK. Chondroitin sulfate proteoglycan immunoreactivity increases following spinal cord injury and transplantation. Exp Neurol 1999, 160: 51-65

3 Zuo J, Neubauer D, Dyess K, Ferguson TA, Muir D. Degradation of chondroitin sulfate proteoglycan enhances the neurite-promoting potential of spinal cord tissue. Exp Neurol 1998; 154: 654-662.

4 Bradbury EJ, Moon LD, Popat RJ, King VR, Bennett GS, Patel PN et al. Chondroitinase $A B C$ promotes functional recovery after spinal cord injury. Nature 2002; 416 636-640.

5 Houle JD, Tom VJ, Mayes D, Wagoner G, Phillips N, Silver J. Combining an autologous peripheral nervous system "bridge" and matrix modification by chondroitinase allows robust, functional regeneration beyond a hemisection lesion of the adult rat spinal cord J Neurosci 2006; 26: 7405-7415.

6 Tester NJ, Howland DR. Chondroitinase ABC improves basic and skilled locomotion in spinal cord injured cats. Exp Neurol 2008; 209: 483-496.

7 Sammons J, Ahmed N, El-Sheemy M, Hassan HT. The role of BMP-6, IL-6, and BMP-4 in mesenchymal stem cell-dependent bone development: effects on osteoblastic differentiation induced by parathyroid hormone and vitamin D(3). Stem Cells Dev 2004; 13: 273-280.
8 Hassan HT, El-Sheemy M. Adult bone-marrow stem cells and their potential in medicine. J R Soc Med 2004; 97: 465-471.

9 Li H, Yu B, Zhang Y, Pan Z, Xu W, Li H. Jagged1 protein enhances the differentiation of mesenchymal stem cells into cardiomyocytes. Biochem Biophys Res Commun 2006; 341: 320-325.

10 Cizkova D, Rosocha J, Vanicky I, Jergova S, Cizek M. Transplants of human mesenchymal stem cells improve functional recovery after spinal cord injury in the rat. Cell Mol Neurobiol 2006; 26: 1167-1180.

11 Barde YA, Edgar D, Thoenen $\mathrm{H}$. Purification of a new neurotrophic factor from mammalian brain. EMBO J 1982; 1: 549-553.

12 Koda M, Hashimoto M, Murakami M, Yoshinaga K, Ikeda O, Yamazaki M et al. Adenovirus vector-mediated in vivo gene transfer of brain-derived neurotrophic factor (BDNF) promotes rubrospinal axonal regeneration and functional recovery after complete transection of the adult rat spinal cord. J Neurotrauma 2004; 21 : 329-337.

13 Tator $\mathrm{CH}$, Koyanagi I. Vascular mechanisms in the pathophysiology of human spinal cord injury. J Neurosurg 1997; 86: 483-492.

14 Wu GJ, Chen WF, Sung CS, Jean YH, Shih CM, Shyu CY et al. Preventive effects of intrathecal methylprednisolone administration on spinal cord ischemia in rats: the role of excitatory amino acid metabolizing systems. Neuroscience 2007; 147: 294-303.

15 Caggiano AO, Zimber MP, Ganguly A, Blight AR, Gruskin EA. Chondroitinase ABCI improves locomotion and bladder function following contusion injury of the rat spinal cord. J Neurotrauma 2005; 22: 226-239.

16 Brazelton TR, Rossi FM, Keshet GI, Blau HM. From marrow to brain: expression of neuronal phenotypes in adult mice. Science (New York, NY) 2000; 290: 1775-1779

17 Lin J, Wang C, Wu Z. [Preliminary study on effects of human brain-derived neurotrophic factor gene-modified bone marrow mesenchymal stem cells by intravenous transplantation on structure and function of rat injured spinal cord]. Zhongguo Xiu Fu Chong Jian Wai Ke Za Zhi 2010; 24: 982-987.

18 Yick LW, Cheung PT, So KF, Wu W. Axonal regeneration of Clarke's neurons beyond the spinal cord injury scar after treatment with chondroitinase ABC. Exp Neurol 2003; 182 160-168.

19 Goto A, Furukawa S. [Experimental changes in BDNF- and NT-3-like immunoreactivities in the spinal cord following its transection]. Nihon Seikeigeka Gakkai Zasshi 1995; 69: 506-516.

20 Tobias CA, Shumsky JS, Shibata M, Tuszynski MH, Fischer I, Tessler A et al. Delayed grafting of BDNF and NT-3 producing fibroblasts into the injured spinal cord stimulates sprouting, partially rescues axotomized red nucleus neurons from loss and atrophy, and provides limited regeneration. Exp Neurol 2003; 184: 97-113.

21 Yu K, Ye X, Qu S. Intraspinal implantation of genetically modified myoblasts with brain derived neurotrophic factor gene in treating spinal cord injury in rats: neurophysiological study. Zhonghua Wai Ke Za Zhi 2001; 39: 724-727.

22 Deng YB, Yuan QT, Liu XG, Liu XL, Liu Y, Liu ZG et al. Functional recovery after rhesus monkey spinal cord injury by transplantation of bone marrow mesenchymal-stem cellderived neurons. Chin Med J 2005; 118: 1533-1541.

23 Sasaki M, Radtke C, Tan AM, Zhao P, Hamada H, Houkin K et al. BDNF-hypersecreting human mesenchymal stem cells promote functional recovery, axonal sprouting, and protection of corticospinal neurons after spinal cord injury. J Neurosc 2009; 29: $14932-14941$.

24 Hong YP, Lee HC, Kim HT. Treadmill exercise after social isolation increases the levels of NGF, BDNF, and synapsin I to induce survival of neurons in the hippocampus, and improves depression-like behavior. J Exerc Nutrition Biochem 2015; 19: 11-18.

25 Saeednia S, Bahadoran H, Amidi F, Asadi MH, Naji M, Fallahi P, Nejad NA. Nerve growth factor in human semen: Effect of nerve growth factor on the normozoospermic men during cryopreservation process. Iran J Basic Med Sci 2015; 18: 292-299. 